This document is the Accepted Manuscript version of a Published Work that appeared in final form in ACS Applied Materials and Interfaces, copyright (c) American Chemical Society after peer review and technical editing by the publisher. To access the final edited and published work see: https://dx.doi.org/10.1021/acsami. 7b15564. 


\section{Surface Functionalization of Metal- Organic Framework Crystals with Catechol Coatings for Enhanced Moisture Tolerance}

Javier Castells-Gil ${ }^{\dagger}$ Fernando Novio ${ }^{\ddagger}$ Natalia M. Padial ${ }^{\dagger}$ Sergio Tatay ${ }^{\dagger}$ Daniel RuízMolina ${ }^{\ddagger}$ Carlos Martí-Gastaldo ${ }^{\star \dagger}$

† Instituto de Ciencia Molecular (ICMol), Universitat de València, Catedrático José Beltrán-2 46980, Paterna, Spain

¥ Catalan Institute of Nanoscience and Nanotechnology (ICN2), CSIC and The Barcelona Institute of Science and Technology, Campus UAB 08193, Bellaterra, Spain

\section{ABSTRACT}

Robust catechol coatings for enhanced moisture tolerance were produced in one step by direct reaction of Hong Kong University of Science and Technology (HKUST) with synthetic catechols. We ascribe the rapid formation of homogeneous coatings around the metal-organic framework particles to the biomimetic catalytic activity of $\mathrm{Cu}$ (II) dimers in the external surface of the crystals. Use of fluorinated catechols results in hydrophobic, permeable coatings that protect HKUST from water degradation while retaining close to $100 \%$ of its original sorption capacity. 


\section{INTRODUCTION}

Metal-organic frameworks (MOFs) are crystalline, molecular solids built from the linking of organic and inorganic components with coordinative bonds. The controllable positioning of inorganic clusters or metal nodes, typically coined secondary building units (SBUs), and organic linkers into the three-dimensional space enables the assembly of topologically regular structures with surface areas up to $8.000 \mathrm{~m} 2 \cdot \mathrm{g}-1$.(1) The combination of internal porosity, adequate thermal stability, and structural/chemical diversities beyond comparison makes them appealing materials for gas storage,(2, 3) separation, $(4,5)$ catalysis(6) and degradation of warfare agents, $(7,8)$ to cite a few. In spite of promising results, the poor water stability of MOFs in humid conditions has been recognized as one of the key limitations for large-scale application.

Prototypical materials such as MOF-5 or Hong Kong University of Science and Technology (HKUST) are prone to degradation with moisture due to the hydrolysis of weak metal-oxo coordination bonds that results in linker replacement with water.(9, 10) This provokes uncontrolled structural changes for a concomitant decrease in porosity after an induction process that becomes more drastic for higher water condensation within the pore.(11) This problem can be solved by direct synthesis of hydrophobic MOFs $(12,13)$ or by endowing the framework with stronger coordination bonds for more robust SBUs by using basic N-donor ligands(14-16) or highly charged metals such as $\mathrm{Zr}$ or Ti(IV).(17-20) However, this approach is limited to new materials and does not permit enhancing the stability against moisture of those already available. This can be instead done by introducing alkyl or fluorinated groups to the aromatic linkers by postsynthetic modification (PSM) or chemical vapor deposition (CVD) treatment. $(21,22)$ Unfortunately, these alternatives also suffer 
from limitations such as the reduction of porosity as a result of the introduction of bulkier substituents in the empty space, complex procedure or sophisticated instrumentation. Alternatively, recent examples demonstrate that MOF crystals can be efficiently protected from hydrolytic degradation by one-step encapsulation in hydrophobic coatings of carbon,(23) polydimethylsiloxane (PDMS)(24) or organic polymers.(25) These examples highlight the importance of finding simple, general alternatives that permit stabilizing water-sensitive MOFs at high humidity levels while preserving their original structure and porosity, preferably at a single crystal level.

Polydopamine (PDA) represents an efficient universal coating to functionalize a broad range of substrates with different nature and dimensions.(26) So far, a few examples of MOF@PDA systems have been reported to enhance the dispersion and separation capabilities of MOFs in mixed matrix membranes(27) and enzyme/metalorganic composites for high-stability and easy reusability.(28) However, the preparation of these coatings is restricted to the polymerization of dopamine in Trisbuffered solutions for slightly basic conditions, susceptible to interfere with the stability of MOFs. Moreover, endowing these coatings with hydrophobicity would involve additional synthetic steps or introducing alkyl/fluorinated chains to PDA, which might result in poor control over functionalization and ineffective protection against moisture. Alternative routes for the one-step synthesis of PDA-like hydrophobic coatings have been also described; however, they involve harsh basic conditions.(26) In this context, Bortoluzzi et al. recently reported that dopamine polymerization in solution can be promoted by a dinuclear $\mathrm{Cu}(\mathrm{II})$ complex featuring a $\mathrm{Cu} 2(\mu-\mathrm{OH})$ central core.(29) This biomimetic system displays catecholase-like catalytic activity reminiscent of natural enzymes such as catechol oxidase(30) and tyrosinase.(31) We argued that MOFs based on the interconnection of related $\mathrm{Cu}$ (II) 
dimers might also facilitate the polymerization of synthetic catechols such as 4heptadecylcatechol (hdcat) and fluorinated 4-undecylcatechol (fdcat). This would allow for producing organic coatings surrounding the MOF crystal in only one step and under very mild conditions while also providing control over its moisture tolerance simply by suitable choice of the chemical functionalities in the catechol alkyl organic chain (Scheme 1).

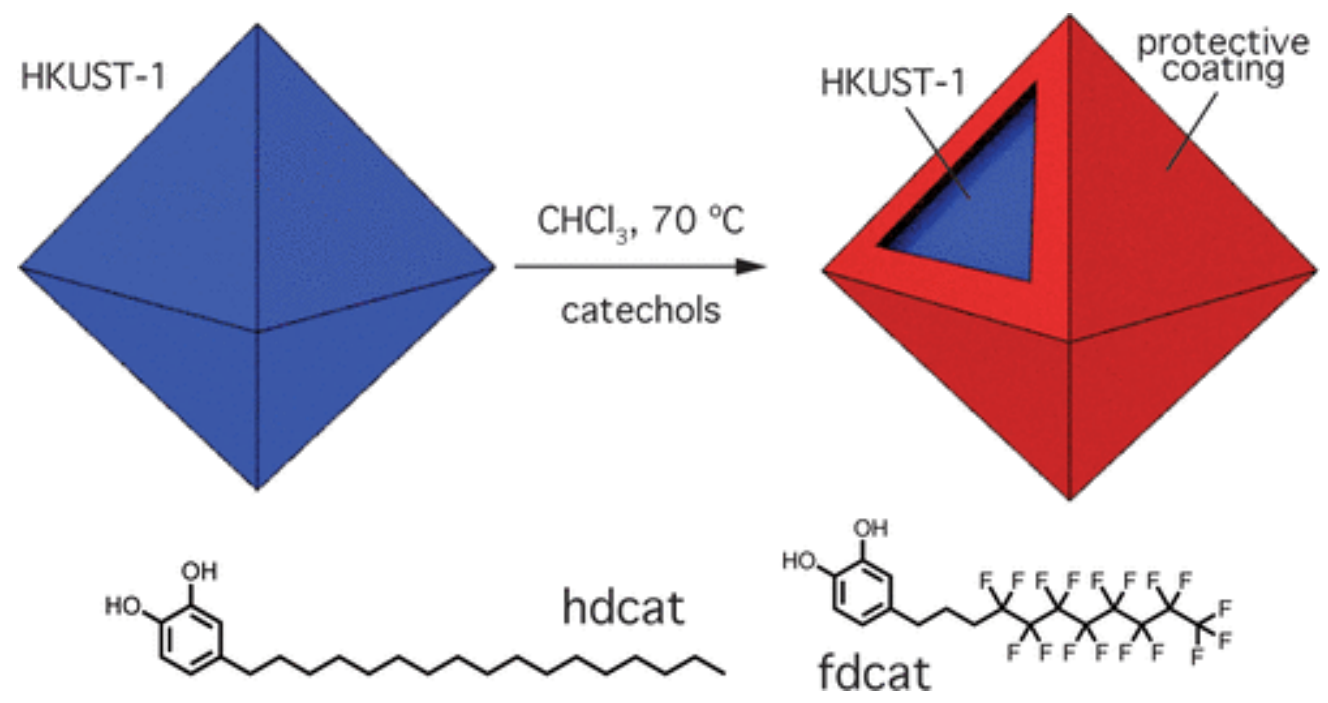

Scheme 1. Formation of Organic Coatings by Direct Reaction of Synthetic Catechols with HKUST Crystals

To validate our approach, we chose HKUST (Cu3(btc)2; btc= 1,3,5benzenetricarboxylate) as an archetypical MOF sensitive to water. It is composed of paddlewheel [Cu2(O2CR)4] units, reminiscent of catechol oxidase catalytic sites, which are bridged by btc ligands to form a porous 3D network.(32) HKUST displays promising features for industrial applications such as solvent-free synthesis, good $\mathrm{CH} 4$ storage at room temperature, easy processing as thin-films or facile fabrication of membranes. However, large-scale application is severely limited by its poor water stability. As confirmed by solid-state NMR, water adsorption isotherms and powder X-ray diffraction (PXRD) studies, exposure of HKUST to water results in a first partial 
collapse $(50 \%$ decrease in porosity) followed by irreversible degradation of the structure after $24 \mathrm{~h}$ to form dense, nonporous phases. $(33,34)$

\section{Results and Discussion}

For the sake of reproducibility, all experiments were performed by using commercially available HKUST (BASOLITE C-300). Our preliminary tests suggested that control over the distribution particle size is important for obtaining reproducible surface density functionalization. The solid provided by the commercial supplier displayed a narrow dispersion close to $50 \mu \mathrm{m}$ (Figure S1), more adequate than the heterogeneity we encountered for the solids prepared by different synthetic routes. Crystals were suspended in separate solutions of hdcat and fdcat $(0.14$ and $0.09 \mathrm{M}$, respectively) in anhydrous chloroform in a glovebox. Differences in concentration were imposed by the different solubility of the catechols. Capped glass vials were taken out and sonicated for a few seconds to produce stable suspensions of the solid. Mixtures were heated at $70{ }^{\circ} \mathrm{C}$ overnight under static conditions to facilitate linker exchange and subsequent polymerization at the surface of the crystals. Successful incorporation of the catechols was first evidenced by the better dispersibility of the crystals in the organic reaction medium. hdcat-HKUST and fdcat-HKUST were then separated by centrifugation, rinsed thoroughly with methanol and chloroform, and left to dry at room temperature inside the glovebox.

Surface modification of the crystals of HKUST was first monitored by Fourier transform infrared (FT-IR) spectroscopy of the solids. hdcat-HKUST features a sharp doublet at 2859 and $2917 \mathrm{~cm}-1$ characteristic of the aliphatic chains in hdcatHKUST, and fdcat-HKUST displays a triplet between 1110 and $1195 \mathrm{~cm}-1$ linked to 
the stretching vibrations of the alkyl fluorinated chain in fdcat, both absent in the original MOF. The broadening of the $\mathrm{O}-\mathrm{H}$ stretching band close to $3500 \mathrm{~cm}-1$ does not permit to rule out the presence of fully protonated linkers in both cases (Figure 1a). Changes in the IR spectra suggest that catechols are grafted to HKUST. We used high resolution XPS for studying the effect of catechol binding at the surface of the crystals. Carbon, oxygen, and copper signals are summarized in Figures S3a-d. Oxygen 1s spectra are dominated by the contribution of HKUST. This is also the case of the $\mathrm{C} 1 \mathrm{~s}$ region, except of fdcat-HKUST, which displays additional peaks at 292 and $294 \mathrm{eV}$ corresponding to -CF2 and -CF3 groups, respectively, in the fluorinated alkyl chain also present in fdcat.(35) $\mathrm{Cu} 2 \mathrm{p}$ spectra displays clear changes linked to the presence of two copper chemical states at the surface of HKUST, $\mathrm{Cu}(\mathrm{I})$ and $\mathrm{Cu}(\mathrm{II})$ (Figure 1b). All solids feature a main $\mathrm{Cu}(2 \mathrm{p} 3 / 2)$ component at $935 \mathrm{eV}$, accompanied by less intense shakeup satellite peaks at higher binding energies that confirm the presence of $\mathrm{Cu}(\mathrm{II})$ species that can be ascribed to bare HKUST.(36) In turn, hdcat-HKUST and fdcat-HKUST feature an additional contribution at $933 \mathrm{eV}$. This is indicative of the presence of $\mathrm{Cu}(\mathrm{I})$ at the surface,(37) likely due to reaction with the catechols involved in the oxidation and subsequent polymerization of hdcat and fdcat. 

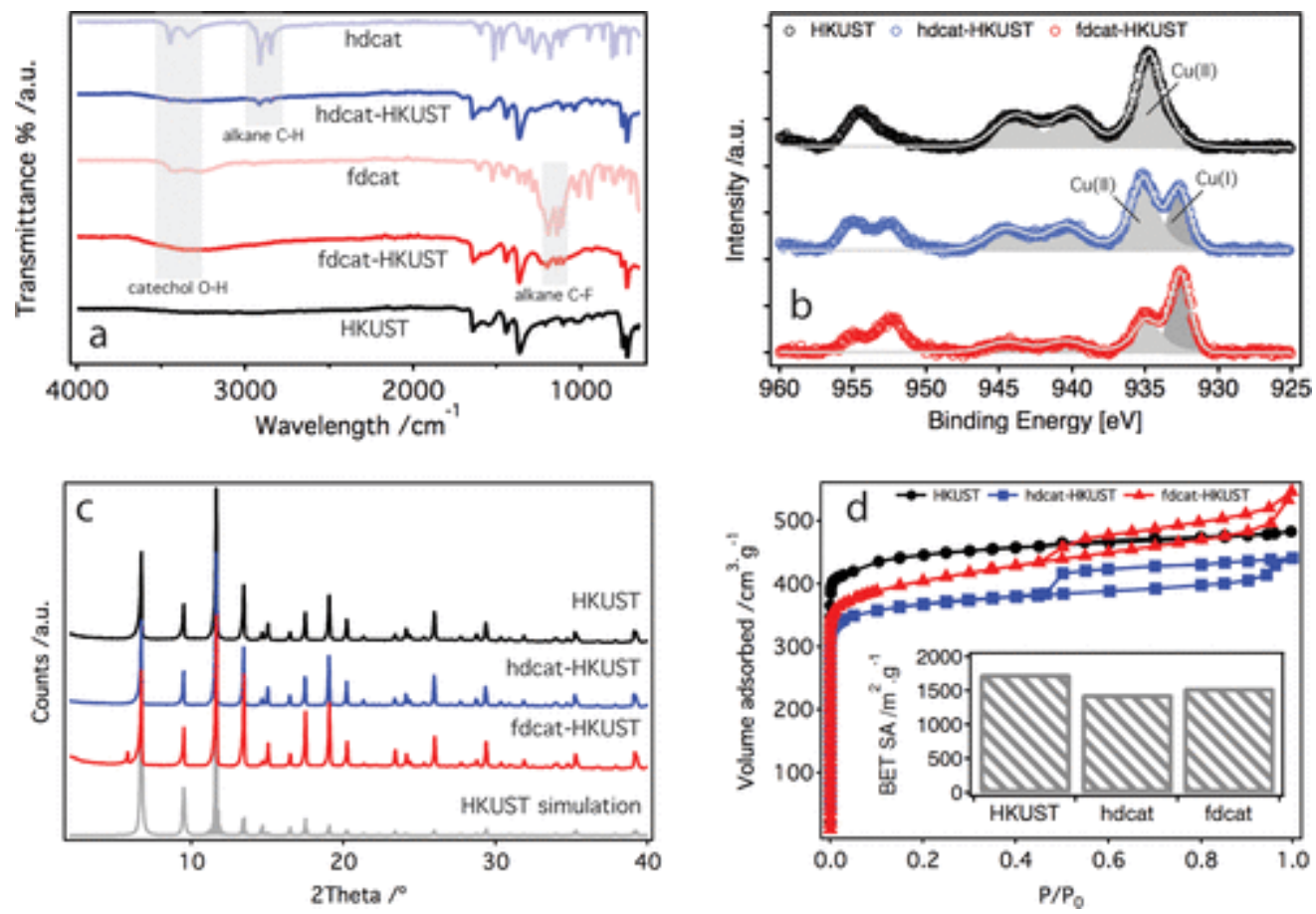

Figure 1. Physical characterization of HKUST crystals after catechol functionalization. (a) FT-IR spectra comparison highlighting the fingerprints that account for the presence of characteristic vibrational modes from the catechol linkers and broadening of the $\mathrm{O}-\mathrm{H}$ stretching band. (b) $\mathrm{Cu} 2 \mathrm{p}$ high-resolution XPS spectra. Dashed areas stand for the deconvolution of the copper signal into $\mathrm{Cu}(\mathrm{I})$ and $\mathrm{Cu}(\mathrm{II})$ upon catechol grafting. See Figures S3a-d for more details. (c) Comparison of the simulated and experimental PXRD patterns of HKUST, hdcat-HKUST, and fdcatHKUST. (d) N2 isotherms and multipoint BET surface areas (inset) of HKUST before and after functionalization. See Table S2 for complete porosity metrics.

PXRD confirms surface modification proceeds with no significant impact over the structure or crystal size (Figures 1c and S4). Thermogravimetric analysis (TGA) is also consistent with minor modification to the structure of HKUST or its thermal stability as all solids decompose close to $300{ }^{\circ} \mathrm{C}$ (Figure S5). By assuming that the 
differences in the weight loss percentage after MOF pyrolysis can be directly ascribed to the incorporation of alkylic catechols, we estimate a weight percentage of 3.6 and $2.1 \%$ for hdcat and fdcat, respectively. Catechol grafting has a minor impact over the porosity of the activated solids, as evaluated by $\mathrm{N} 2$ adsorption-desorption measurements at $77 \mathrm{~K}$ (Figure 1d). The original type-I isotherm of HKUST is retained for hdcat-HKUST and fdcat HKUST, which in addition display hysteretic gas uptake at high relative pressures. This is likely due to kinetic limitations for gas diffusion imposed by the permeable but nonporous organic layers that protect the crystals. Multipoint Brunauer-Emmett-Teller (BET) values show a decrease in surface area from $1756 \mathrm{~m} 2 \cdot \mathrm{g}-1$ for HKUST to $1461 \mathrm{~m} 2 \cdot \mathrm{g}-1$ (83\%) in hdcat-HKUST and 1550 $\mathrm{m} 2 \cdot \mathrm{g}-1$ (88\%) in fdcat-HKUST. This reduction is somewhat above the expected according to the percentage of catechols incorporated as estimated from TGA data (Figure S5) and might be also influenced by partial blocking of the pore entrance by the manifold conformational rotation of alkylic chains. A similar behavior was described for the modification of HKUST with perfluorohexane.(21) Micropore volume calculations with the Dubinin-Radushkevich (DR) equation also confirm preservation of the original porosity (Table S2). All solids display a narrow distribution of well-defined micropores with volumes ranging from 0.65 to 0.55 $\mathrm{cm} 3 \cdot \mathrm{g}-1$

Scanning electron microscopy (SEM) imaging of HKUST before and after ligand exchange reaction further confirms that functionalization is respectful with the micrometric size and octahedral morphology of the crystals (Figure 2, Table S2). Whereas unmodified HKUST displays smooth faces, catechol grafting results in the formation of a homogeneous, corrugated layer covering the crystals in hdcatHKUST and fdcat-HKUST. The presence of fluorine in the last allowed for verifying 
the uniformity of the coating by energy dispersive X-ray (EDX) line profile and pointscan analysis of the sample. Figure S6 suggests a homogeneous distribution of all elements with a $\mathrm{F} / \mathrm{Cu}$ ratio of $2.2 \pm 0.3 \%$, consistent with the coating density estimated from TGA data. Exposure of these crystals to longer irradiation times at higher beam intensities $(20 \mathrm{kV})$ results in complete degradation of the external layer, which further confirms its organic nature (Figure S7). We also treated a dispersion of the crystals in organic solvent with ultrasounds for testing the strength of the grafting. Except for residual sections that display partial fracture, the superficial layer remains tightly bound to the crystals, as expected for the formation of coordination bonds with the metal nodes at the surface of HKUST, that seems to provide a robust support for the cross-linking of additional catechol units. This agrees well with close examination of a handful of areas after peeling off the coating for a rough estimate of the thickness of the outer layer, which lies around $640 \pm 100 \mathrm{~nm}$ (Figure S8). This is substantially thicker than related catechol coatings,(26) thereby suggesting a very efficient polymerization at the surface of the crystals. However, we cannot rule out partial leaching of $\mathrm{Cu}(\mathrm{II})$ ions from the crystal surface into the solid-liquid interface by catechol coordination that might also favor polymerization. Catechol polymerization is generally driven by self-oxidation under aerobic conditions in basic medium. In contrast, we observe the formation of thick, homogeneous coatings in oxygen-free conditions without the addition of an auxiliary base. This seems to sustain the ability of HKUST to facilitate the oxidation of the catechol groups anchored to the surface. As a tentative mechanism, we propose the initial coordination of catechols to the $\mathrm{Cu}(\mathrm{II})$ paddlewheel units terminated by btc linkers located at the $\{111\}$ crystallographic faces in the surface of the crystals.(38) This process can take place by linker exchange reactions without disrupting the 
underlying connectivity in the framework, as previously demonstrated by Kitagawa and coworkers.(39) Next, copper reduction would take place as result of the electron transfer coupled to the oxidation of the catechol ring into o-quinone form, which in turn would react with unoxidized catechol units to produce highly reactive semiquinone radicals via comproportionation.(31) The radicals formed might then undergo aryloxy radical coupling to form covalent cross-links between adjacent catechol rings akin to those in polydopamine. A similar cross-linking pathway was reported for mussel adhesive proteins.(40) Polymer formation is confirmed by $1 \mathrm{H}$ NMR of hdcat-HKUST crystals in $\mathrm{CDCl} 3$ after digestion with an aqueous solution of Na2S. NMR spectrum (Figure S14a) is consistent with that reported by Ruiz-Molina et al.(26) for polymerization of catechols in basic medium. To prove further the role played by copper in the polymerization reaction, we treated hdcat with and without $\mathrm{Cu}(\mathrm{OAc}) 2$ in chloroform under polymerization conditions. As expected, NMR measurements confirm that hdcat remains unchanged in absence of copper, whereas it does undergo polymerization in the presence of $\mathrm{Cu}(\mathrm{II})$ cations (Figure S14b). We were also intrigued on the specificity of copper in directing catechol polymerization. If this could be extended to other metal ions, this methodology might be regarded as a versatile route for endowing other archetypal MOFs such as MOF5 with enhanced water stability. In this regard, we performed equivalent reactions in the presence of $\mathrm{Zn}(\mathrm{OAc}) 2$. However, NMR rules out polymerization, confirming that this type of reactivity is specific to $\mathrm{Cu}(\mathrm{II})$ ions. 

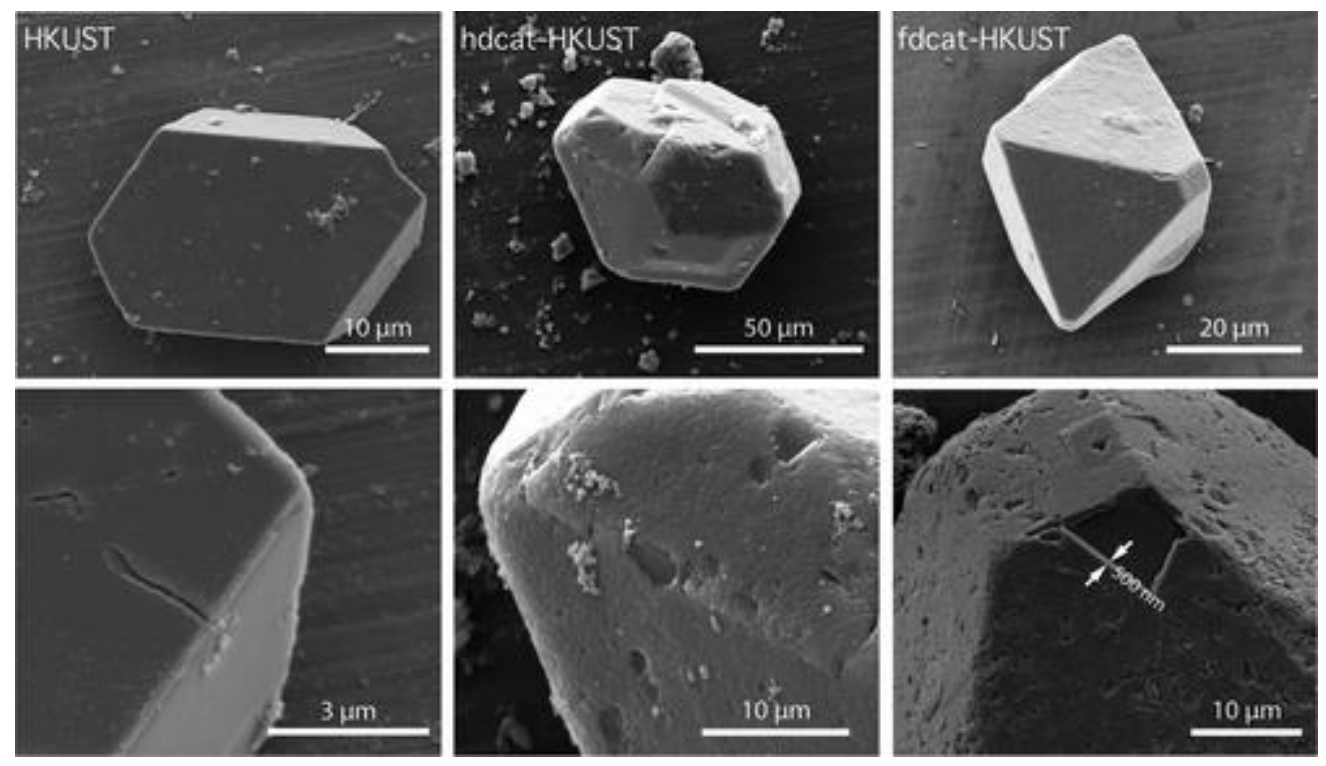

Figure 2. SEM imaging of functionalized HKUST crystals. Solids retain the original size and morphology but display a homogeneous, submicrometric outer layer after one-step grafting of synthetic catechols. This is more clearly seen for the zoom-in in the bottom panel.

Differential behaviors of the solids in contact with water solution provide further proof for the efficient coating of the particles. Bare HKUST immediately sinks in solution, while hdcat-HKUST and fdcat-HKUST float on water (Figure 3a). This can be correlated with changes in the contact angle (CA) values for all solids. Unmodified HKUST displays a CA close to $0^{\circ}$, characteristic of hydrophilic materials, whereas surface functionalization with hdcat and fdcat results in CA values of 107 and $124 \pm$ $1^{\circ}$, indicative of hydrophobic solids (Figure 3b). Relative differences can be ascribed to the increased hydrophobicity of fluorinated alkyl chains in fdcat compared to those of alkyl hdcat. These values agree well with those reported for coordination polymer nanoparticles and substrates modified with related catechols.(26, 41) Though alternative routes such as postsynthetic modification of MIL-53(AI) with fluorinated 
linkers(22) or engineering of external surface corrugation can yield superhydrophobic $\left(\geq 150^{\circ}\right)$ MOFs,(12) modification of the crystal surface with catechols renders hydrophobicity similar to that achieved by simpler methodologies such as one-step encapsulation with PDMS (ca. $\left.130^{\circ}\right)$.(24) Pristine and modified HKUST materials were also immersed in water for three days at room temperature to investigate their tolerance against moisture. As summarized in Figure 4, we studied changes in the structure, particle morphology, and porosity of all samples with PXRD, SEM and N2 adsorption. Compared to the smooth surface of as-made crystals, SEM images of HKUST display signatures of partial degradation with cracks, cavities, and a more corrugated surface as result of water attack (Figures 4a and S9). Changes are even more severe for hdcat-HKUST. Notwithstanding the hydrophobic coating, water exposure triggers the formation of longitudinal needles that cover the surface of the crystals completely (Figure 4b). A closer look reveals that needles grow directly on top of the MOF particles, which seem to be feeding copper to the catechol in the surface acting as a scavenger. This results in flat, micrometric long needles with a lateral length close to $400 \mathrm{~nm}$ that display a uniform distribution of $\mathrm{Cu}, \mathrm{C}$ and $\mathrm{O}$ (Figure S11 and S12). We performed additional experiments to investigate the formation of the needles. Room temperature reaction of hdcat with an aqueous solution of $\mathrm{Cu}(\mathrm{OAc}) 2(0.1 \mathrm{M})$ in water in open air drives the formation of a fibrous polymeric matrix rich in copper (Figure S13). 


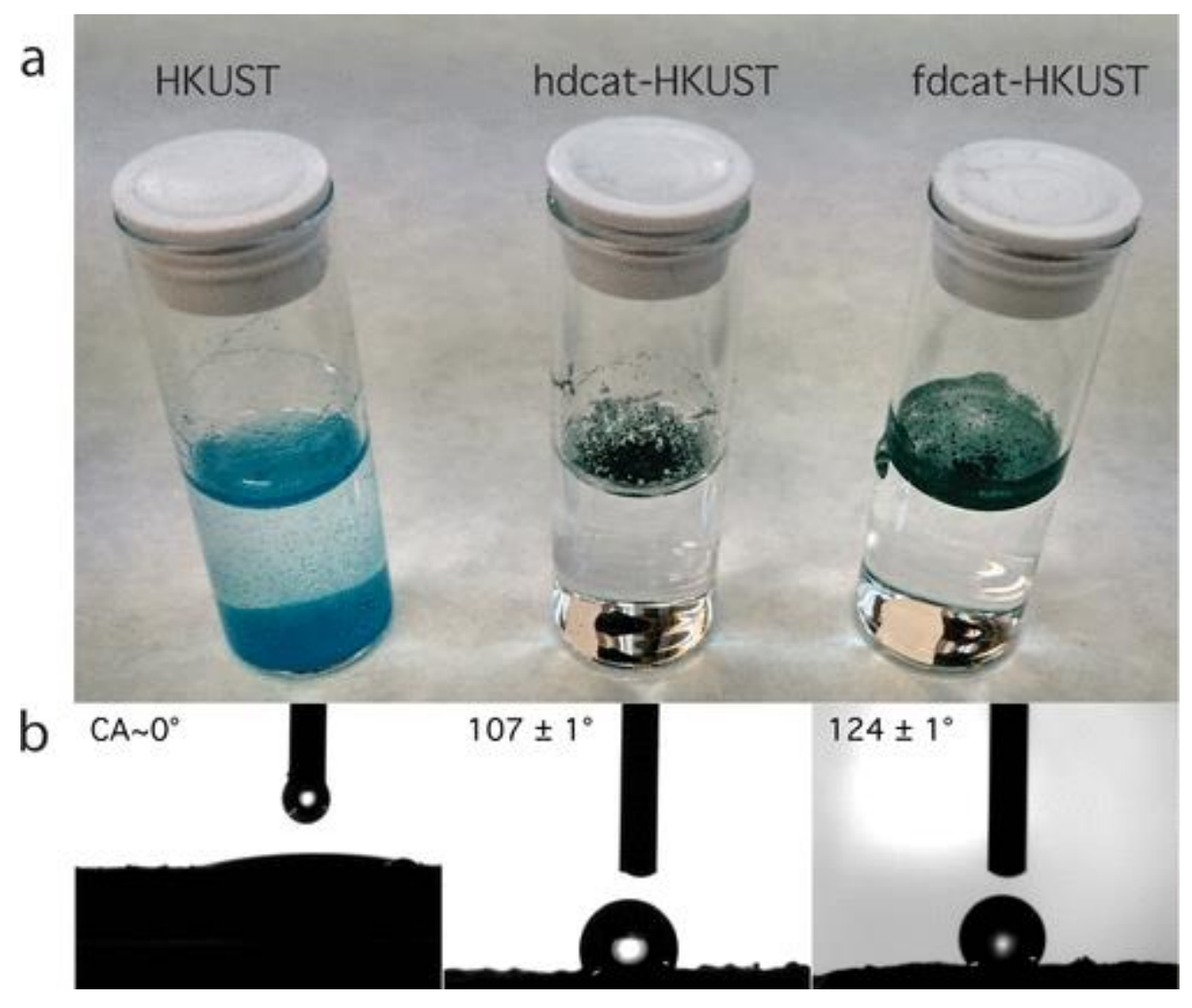

Figure 3. Compared to bare HKUST, modified particles float in water (a) as result of increasing hydrophobicity according to their contact angle values (b). 

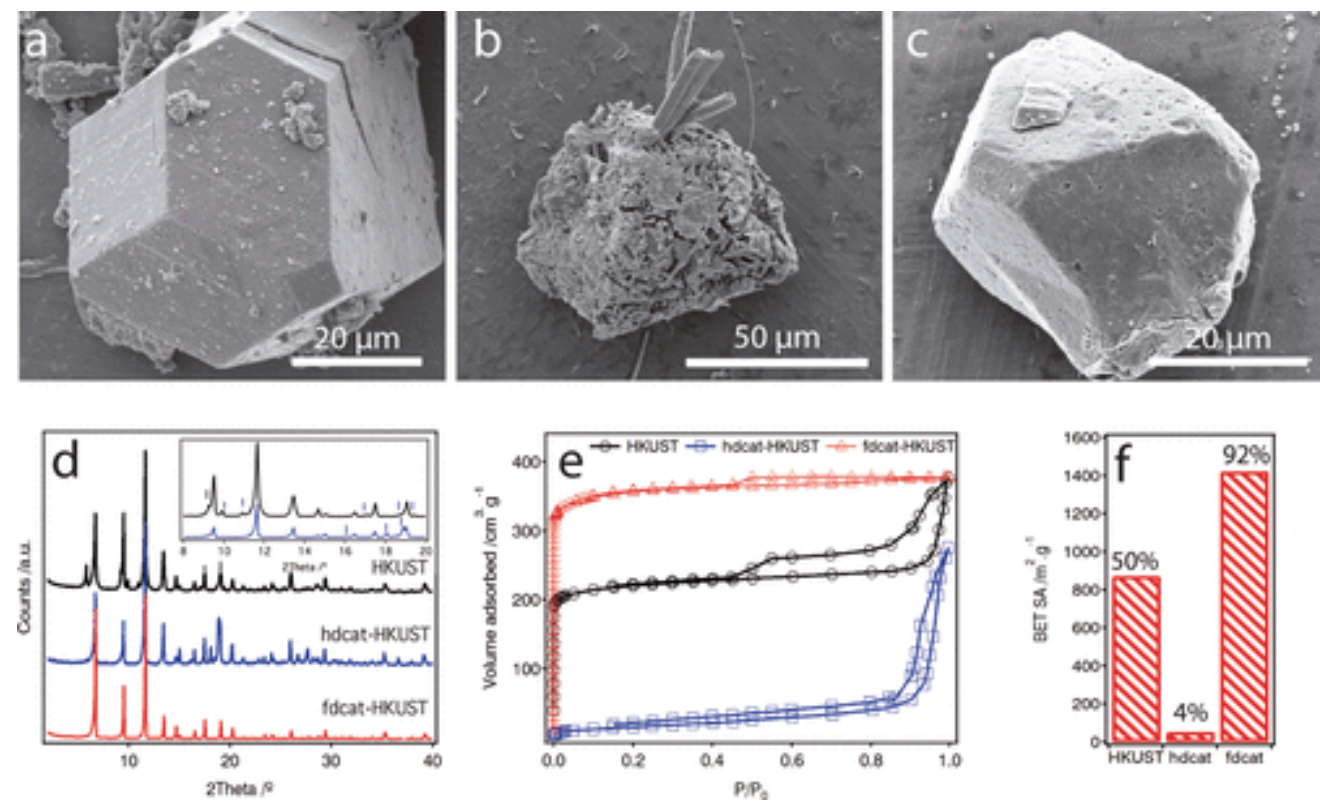

Figure 4. Morphology, structure and porosity of functionalized HKUST crystals after digestion in water. SEM pictures of HKUST (a), hdcat-HKUST (b), and fdcatHKUST (c) after soaking of the solids in water for 3 days. (d) PXRD of the solids after water treatment after 3 days (7 days for fdcat-HKUST). Inset highlights the changes in the diffraction pattern of HKUST and hdcat-HKUST that cannot be indexed for the original phase. See Figure S4 for data refinement. (e) N2 isotherms and (f) percentage values for the retained porosity with the respect to as-made materials before exposure to water. See Table S2 for complete porosity metrics.

We argue that preorganization of the catechol coating around the crystal coupled to slow leaching from the MOF when exposed to water controls the nucleation and growth of the resulting compound for the formation of different morphologies. Surface alkyl catechol molecules do not provide an efficient protective shell but enable partial infiltration of water for a permeable coating. In turn, increased hydrophobicity of alkyl fluorinated catechols in fdcat-HKUST is quite effective in 
avoiding degradation as crystals retain the original morphology and do not display changes in the structure of the organic shell surrounding the crystal (Figure 4c). Water practically does not affect its chemical integrity as the $\mathrm{F} / \mathrm{Cu}$ ratio of $1.9 \pm$ $0.4 \%$, according to EDX point-scan mapping after 3 days of incubation in water (Figure S15), remains close to the original value. PXRD of HKUST confirms partial degradation with water, featuring additional peaks at 9.2, 9.9 and $19.5^{\circ}$ (inset Figure 4d) that could not be straightforwardly assigned to other hydrated crystalline phases reported for Cu-btc MOFs. These diffraction lines are not present in hdcat-HKUST, which displays only new signals of weak intensity at 18.1 and $18.9^{\circ}$ that do not correspond to the same phase from degradation of HKUST and are more likely associated with in situ formation of poorly diffracting needles as a contaminant phase. Degradation also results in partial decrease of diffraction intensity with respect to that of fdcat-HKUST that remains instead unchanged with no signature of phase segregation or amorphization. To test further the stability of fdcat-HKUST, we extended the incubation time in water to 1 week with no significant decrease in crystallinity. Changes in the tolerance to water after catechol functionalization are also reflected in the sorption capability of the solids after water treatment. Compared to bare HKUST, which loses $50 \%$ of the original porosity (SBET $=874 \mathrm{~m} 2 \cdot \mathrm{g}-1$ ), degradation is more severe in hdcat-HKUST, which retains only $4 \%$ of the porosity of the solid before soaking in water (55 m2.g-1). For hdcat-HKUST, catechol functionalization has a negative effect over moisture tolerance, as it does not improve it but instead accelerates the decomposition process due to water permeability and increased reactivity of metal ions in the surface of the crystals. In turn, fdcat-HKUST features a robust coverage that retains $92 \%$ of the original porosity (1550 m2.g-1) and a total micropore volume $0.58 \mathrm{~cm} 3 \cdot \mathrm{g}-1$ even after 1 
week in water. This is likely due to the enhanced hydrophobicity provided by the fluorinated alkyl chain that repels water molecules more effectively.

\section{Conclusions}

In summary, we report the ability of HKUST to induce the formation of polydopamine-like coatings by direct reaction with synthetic catechols in anaerobic conditions without base addition. Compared to other methodologies, this route enables rapid formation of robust coatings in only one step regardless of the lateral substitution of the alkyl chain in the catechol. We ascribe this behavior to the external surface of HKUST, rich in potentially catalytic $\mathrm{Cu}$ (II) dimers terminated by btc linkers capable of incorporating catechol units by linker exchange reactions at mild temperatures.(39) Reminiscent of the enzymatic activity of catechol oxidase,(30) this would assist one electron oxidation into o-quinone followed by cross-link polymer formation. As result, MOF particles can be straightforwardly protected with a thick, homogeneous, organic shell at a single crystal level. This approach also provides control over the function of the protective shell as it can be engineered by suitable choice of the synthetic catechols. By using alkyl fluorinated derivatives, we achieved gas permeable coatings that show efficient hydrophobic protection for HKUST against water degradation and retain its original sorption capacity. We are confident the versatility of this approach will provide an interesting avenue toward the development of functional coatings for the adsorption of chiral molecules or volatile organic compounds by fine-tuning of their interactions with the lateral chains of related synthetic catechols. 


\section{Experimental Section}

\section{Materials and Reagents}

Commercial Basolite C300 and anhydrous solvents were purchased from SigmaAldrich and used as received. Ultrapure water from Milli-Q equipment was used for water stability tests when required. All reagents and solvents were kept inside the glovebox to avoid any contact with humid environments prior to catechol coating that might drive competing polymerization reactions. All reagents were used without any previous purification. hdcat and fdcat were synthesized according to the reported procedure. $(26,42)$

\section{Synthesis of hdcat-HKUST}

Fifty milligrams of hdcat $(0.14 \mathrm{mmol})$ were dissolved in $1 \mathrm{~mL}$ of anhydrous $\mathrm{CHCl} 3$ in a rubber-lined capped glass vial, followed by addition of $10 \mathrm{mg}$ of HKUST to the solution. The resultant mixture was sonicated for a few minutes to produce a homogeneous suspension and heated at $70{ }^{\circ} \mathrm{C}$ overnight. The coated material was separated by centrifugation, washed thoroughly in two steps with anhydrous $\mathrm{CH} 3 \mathrm{OH}$ and $\mathrm{CHCl} 3$, and left to dry at room temperature in the glovebox. The coated material was stored in the glovebox, and samples were taken out for analysis, NMR measurements, and water treatment.

\section{Synthesis of fdcat-HKUST}

fdcat derivative was prepared by following the same procedure but dissolving $50 \mathrm{mg}$ $(88 \mu \mathrm{mol})$ of the synthetic cathecol in $1 \mathrm{~mL}$ of anhydrous $\mathrm{CHCl} 3$, followed by addition of $10 \mathrm{mg}$ of HKUST to the solution. Washing, drying, and handling of the sample was the same as described above. 


\section{Physical and Chemical Characterization}

Infrared spectra were recorded in an Agilent Cary 630 FTIR spectrometer equipped with an ATR module. Thermogravimetric analysis was carried out with a Mettler Toledo TGA/SDTA 851 between 25 and $800{ }^{\circ} \mathrm{C}$ at a rate of $5{ }^{\circ} \mathrm{C}$ min-1 with a flow of N2:O2 (4:1). PXRD patterns were collected in a PANalytical X'Pert Pro diffractometer using copper radiation $(\mathrm{Cu} \mathrm{Ka}=1.5418 \AA)$ with an X'Celerator detector operating at $40 \mathrm{~mA}$ and $45 \mathrm{kV}$. Profiles were collected in the $2^{\circ}<2 \theta<40^{\circ}$ range with a step size of 0.013 . Particle morphologies and dimensions were studied with a FEI Quanta 650 FEG scanning electron microscope operating between 2 and $5 \mathrm{kV}$ over nonmetalized samples or between 5 and $15 \mathrm{kV}$ over metallized samples. EDX line-scan and mappings were performed using the same apparatus at accelerating voltage $20 \mathrm{kV}$ over nonmetallized samples. Surface area, pore size, and volume values were calculated from nitrogen adsorption-desorption isotherms recorded at $77 \mathrm{~K}$ on an AUTOSORB-6 apparatus. Samples were degassed for $4 \mathrm{~h}$ at $170^{\circ} \mathrm{C}$ and $10-6$ Torr prior to analysis. Surface areas were estimated according to the BET model, and pore size dimensions were calculated by the quenched solid density functional theory model (QSDFT) for the adsorption branch assuming a cylindrical pore model. XPS was performed at the X-ray Unit of the Universitat d'Alacant using a K-Alpha X-ray photoelectron spectrometer system (Thermo Scientific). All spectra were collected using Al Ka radiation (1486.6 eV), monochromatized by a twin crystal monochromator, yielding a focused X-ray spot (elliptical in shape with a major axis length of $400 \mu \mathrm{m}$ ) at $3 \mathrm{mAC}$ and $12 \mathrm{kV}$. The alpha hemispherical analyzer was operated in the constant energy mode with survey scan pass energies of $200 \mathrm{eV}$ to measure the whole energy band and $50 \mathrm{eV}$ in a narrow scan to selectively measure the particular elements. XPS data were analyzed 
with Avantage software. A smart background function was used to approximate the experimental backgrounds. Charge compensation was achieved with the system flood gun that provides low energy electrons and low energy argon ions from a single source. NMR spectra were run on a Bruker DRX500 spectrometer. See the Supporting Information for additional experimental details on digestion of copper containing solids for NMR study.

\section{Acknowledgment}

This work was supported by the EU (ERC Stg Chem-fs-MOF 714122), Spanish MINECO (Unit of Excellence María de Maeztu MDM-2015-0538), and the Generalitat

Valenciana (Grant GV/2016/137). C.M.-G. and J.C.-G. thank the Spanish MINECO for a Ramón y Cajal Fellowship and FPI Scholarship (CTQ2014-59209-P), respectively. N.M.P. thanks the Junta de Andalucía for postdoctoral fellowship P10FQM-6050. F.N. and D.R.M. are also grateful to the financial support offered by Project MAT2015-70615-R from the Spanish Government and by FEDER funds. The ICN2 is funded by the CERCA programme/Generalitat de Catalunya and supported by the Severo Ochoa programme of the Spanish Ministry of Economy, Industry and Competitiveness (MINECO, grant no. SEV-2013-0295). 


\section{References}

1.Furukawa, H.; Cordova, K. E.; O'Keeffe, M.; Yaghi, O. M. The Chemistry and Applications of Metal-Organic Frameworks Science 2013, 341 (6149) 1230444DOI: 10.1126/science. 1230444

2.Krause, S.; Bon, V.; Senkovska, I.; Stoeck, U.; Wallacher, D.; Többens, D. M.; Zander, S.; Pillai, R. S.; Maurin, G.; Coudert, F.-X.; Kaskel, S. A Pressure-Amplifying Framework Material with Negative Gas Adsorption Transitions Nature 2016, 532, 348-352DOI: 10.1038/nature17430

3.Mason, J. A.; Oktawiec, J.; Taylor, M. K.; Hudson, M. R.; Rodriguez, J.; Bachman, J. E.; Gonzalez, M. I.; Cervellino, A.; Guagliardi, A.; Brown, C. M.; Llewellyn, P. L.; Masciocchi, N.; Long, J. R. Nature 2015, 527, 357DOI: 10.1038/nature15732

4.Cadiau, A.; Adil, K.; Bhatt, P. M.; Belmabkhout, Y.; Eddaoudi, M. A Metal-Organic Framework-based Splitter for Separating Propylene from Propane Science 2016, 353, 137- 140DOI: 10.1126/science.aaf6323

5.Banerjee, D.; Simon, C. M.; Plonka, A. M.; Motkuri, R. K.; Liu, J.; Chen, X.; Smit, B.; Parise, J. B.; Haranczyk, M.; Thallapally, P. K. Metal-organic Framework with Optimally Selective Xenon Adsorption and Separation Nat. Commun. 2016, 7, 11831DOI: 10.1038/ncomms11831

6.Zhao, M.; Yuan, K.; Wang, Y.; Li, G.; Guo, J.; Gu, L.; Hu, W.; Zhao, H.; Tang, Z. Metal-organic Frameworks as Selectivity Regulators for Hydrogenation Reactions Nature 2016, 539, 76DOI: 10.1038/nature19763

7.Mondloch, J. E.; Katz, M. J.; Isley, W. C., III; Ghosh, P.; Liao, P.; Bury, W.; Wagner, G. W.; Hall, M. G.; DeCoste, J. B.; Peterson, G. W.; Snurr, R. Q.; Cramer, C. J.; Hupp, J. T.; Farha, O. K. Destruction of Chemical Warfare Agents Using Metal-organic Frameworks Nat. Mater. 2015, 14 (5) 512- 516DOI: $10.1038 /$ nmat4238

8.López-Maya, E.; Montoro, C.; Rodríguez-Albelo, L. M.; Aznar Cervantes, S. D.; Lozano-Pérez, A. A.; Cenís, J. L.; Barea, E.; Navarro, J. A. R. Textile/Metal-OrganicFramework Composites as Self-Detoxifying Filters for Chemical-Warfare Agents Angew. Chem., Int. Ed. 2015, 54 (23) 6790-6794DOI: 10.1002/anie.201502094

9.Bellarosa, L.; Castillo, J. M.; Vlugt, T.; Calero, S.; López, N. On the Mechanism Behind the Instability of Isoreticular Metal-Organic Frameworks (IRMOFs) in Humid Environments Chem. - Eur. J. 2012, 18 (39) 12260- 12266DOI: 10.1002/chem.201201212

10.Low, J. J.; Benin, A. I.; Jakubczak, P.; Abrahamian, J. F.; Faheem, S. A.; Willis, R. R. Virtual High Throughput Screening Confirmed Experimentally: Porous Coordination Polymer Hydration J. Am. Chem. Soc. 2009, 131 (43) 1583415842DOI: $10.1021 / \mathrm{ja9061344}$

11.Guo, P.; Dutta, D.; Wong-Foy, A. G.; Gidley, D. W.; Matzger, A. J. Water Sensitivity in Zn4O-Based MOFs Is Structure and History Dependent J. Am. Chem. Soc. 2015, 137 (7) 2651- 2657DOI: 10.1021/ja512382f 
12.Rao, K. P.; Higuchi, M.; Sumida, K.; Furukawa, S.; Duan, J.; Kitagawa, S. Design of Superhydrophobic Porous Coordination Polymers through the Introduction of External Surface Corrugation by the Use of an Aromatic Hydrocarbon Building Unit Angew. Chem., Int. Ed. 2014, 53 (31) 8225- 8230DOI: 10.1002/anie.201404306

13.Yang, C.; Kaipa, U.; Mather, Q. Z.; Wang, X.; Nesterov, V.; Venero, A. F.; Omary, M. A. Fluorous Metal-Organic Frameworks with Superior Adsorption and Hydrophobic Properties toward Oil Spill Cleanup and Hydrocarbon Storage J. Am. Chem. Soc. 2011, 133 (45) 18094- 18097DOI: 10.1021/ja208408n

14.Park, K. S.; Ni, Z.; Côté, A. P.; Choi, J. Y.; Huang, R.; Uribe-Romo, F. J.; Chae, H. K.; O'Keeffe, M.; Yaghi, O. M. Exceptional Chemical and Thermal Stability of Zeolitic Imidazolate Frameworks Proc. Natl. Acad. Sci. U. S. A. 2006, 103 (27) 10186- 10191DOI: 10.1073/pnas.0602439103

15.Colombo, V.; Galli, S.; Choi, H. J.; Han, G. D.; Maspero, A.; Palmisano, G.; Masciocchi, N.; Long, J. R. High Thermal and Chemical Stability in PyrazolateBridged Metal-organic Frameworks with Exposed Metal Sites Chem. Sci. 2011, 2 (7) 1311DOI: 10.1039/c1sc00136a

16.Wang, K.; Lv, X. L.; Feng, D.; Li, J.; Chen, S.; Sun, J.; Song, L.; Xie, Y.; Li, J. R.; Zhou, H. C. Pyrazolate-Based Porphyrinic Metal-Organic Framework with Extraordinary Base-Resistance J. Am. Chem. Soc. 2016, 138 (3) 914- 919DOI: 10.1021/jacs.5b10881

17.Cavka, J. H.; Jakobsen, S.; Olsbye, U.; Guillou, N.; Lamberti, C.; Bordiga, S.; Lillerud, K. P. A New Zirconium Inorganic Building Brick Forming Metal Organic Frameworks with Exceptional Stability J. Am. Chem. Soc. 2008, 130 (42) 1385013851DOI: $10.1021 / \mathrm{ja} 8057953$

18.Feng, D.; Gu, Z. Y.; Li, J. R.; Jiang, H. L.; Wei, Z.; Zhou, H. C. ZirconiumMetalloporphyrin PCN-222: Mesoporous Metal-Organic Frameworks with Ultrahigh Stability as Biomimetic Catalysts Angew. Chem., Int. Ed. 2012, 51 (41) 1030710310DOI: 10.1002/anie.201204475

19.Guillerm, V.; Ragon, F.; Dan-Hardi, M.; Devic, T.; Vishnuvarthan, M.; Campo, B.; Vimont, A.; Clet, G.; Yang, Q.; Maurin, G.; Férey, G.; Vittadini, A.; Gross, S.; Serre, C. A Series of Isoreticular, Highly Stable, Porous Zirconium Oxide Based MetalOrganic Frameworks Angew. Chem., Int. Ed. 2012, 51 (37) 9267- 9271DOI: 10.1002/anie.201204806

20.Devic, T.; Serre, C. High Valence 3p and Transition Metal Based MOFs Chem. Soc. Rev. 2014, 43 (43) 6097-6115DOI: 10.1039/C4CS00081A

21.Decoste, J. B.; Peterson, G. W.; Smith, M. W.; Stone, C. A.; Willis, C. R. Enhanced Stability of Cu-BTC MOF via Perfluorohexane Plasma-Enhanced Chemical Vapor Deposition J. Am. Chem. Soc. 2012, 134 (3) 1486- 1489DOI: 10.1021/ja211182m

22.Nguyen, J. G.; Cohen, S. M. Moisture-Resistant and Superhydrophobic MetalOrganic Frameworks Obtained via Postsynthetic Modification J. Am. Chem. Soc. 2010, 132 (13) 4560- 4561DOI: 10.1021/ja100900c 
23.Yang, S. J.; Park, C. R. Preparation of Highly Moisture-Resistant Black-Colored Metal Organic Frameworks Adv. Mater. 2012, 24 (29) 4010- 4013DOI: 10.1002/adma.201200790

24.Zhang, W.; Hu, Y.; Ge, J.; Jiang, H. L.; Yu, S. H. A Facile and General Coating Approach to Moisture/water-Resistant Metal-Organic Frameworks with Intact Porosity J. Am. Chem. Soc. 2014, 136 (49) 16978- 16981DOI: 10.1021/ja509960n

25.Carné-Sánchez, A.; Stylianou, K. C.; Carbonell, C.; Naderi, M.; Imaz, I.; Maspoch, D. Protecting Metal-Organic Framework Crystals from Hydrolytic Degradation by Spray-Dry Encapsulating Them into Polystyrene Microspheres Adv. Mater. 2015, 27 (5) 869- 873DOI: 10.1002/adma.201403827

26.Saiz-Poseu, J.; Sedó, J.; García, B.; Benaiges, C.; Parella, T.; Alibés, R.; Hernando, J.; Busqué, F.; Ruiz-Molina, D. Versatile Nanostructured Materials via Direct Reaction of Functionalized Catechols Adv. Mater. 2013, 25 (14) 20662070DOI: $10.1002 /$ adma.201204383

27.Wang, Z.; Wang, D.; Zhang, S.; Hu, L.; Jin, J. Interfacial Design of Mixed Matrix Membranes for Improved Gas Separation Performance Adv. Mater. 2016, 28 (17) 3399-3405DOI: 10.1002/adma.201504982

28.Wu, X.; Yang, C.; Ge, J.; Liu, Z. Polydopamine Tethered Enzyme/metal-Organic Framework Composites with High Stability and Reusability Nanoscale 2015, 7 (45) 18883DOI: 10.1039/C5NR05190H

29.de Oliveira, J. A. F.; da Silva, M. P.; de Souza, B.; Camargo, T. P.; Szpoganicz, B.; Neves, A.; Bortoluzzi, A. J. Dopamine Polymerization Promoted by a Catecholase Biomimetic $\mathrm{Cu}(\mathrm{II})(\mu-\mathrm{OH}) \mathrm{Cu}(\mathrm{II})$ complex Containing a Triazine-Based Ligand Dalt. Trans. 2016, 45 (39) 15294- 15297DOI: 10.1039/C6DT02032A

30.Koval, I. A.; Gamez, P.; Belle, C.; Selmeczi, K.; Reedijk, J. Synthetic Models of the Active Site of Catechol Oxidase: Mechanistic Studies Chem. Soc. Rev. 2006, 35 (9) 814DOI: $10.1039 / \mathrm{b} 516250 \mathrm{p}$

31.Yang, J.; Cohen Stuart, M. A.; Kamperman, M. Jack of All Trades: Versatile Catechol Crosslinking Mechanisms Chem. Soc. Rev. 2014, 43 (43) 8271- 8298DOI: 10.1039/C4CS00185K

32.Chui, S.; Lo, S.; Charmant, J.; Orpen, A. G. A Chemically Functionalizable Nanoporous Material [Cu3(TMA)2(H2O)3]n Science 1999, 283 (5405) 11481150DOI: 10.1126/science.283.5405.1148

33.Gul-E-Noor, F.; Michel, D.; Krautscheid, H.; Haase, J.; Bertmer, M. Time Dependent Water Uptake in Cu3(btc)2 MOF: Identification of Different Water Adsorption States by 1H MAS NMR Microporous Mesoporous Mater. 2013, 180, 8 13DOI: 10.1016/j.micromeso.2013.06.033

34.Küsgens, P.; Rose, M.; Senkovska, I.; Fröde, H.; Henschel, A.; Siegle, S.; Kaskel, $\mathrm{S}$. Characterization of Metal-Organic Frameworks by Water Adsorption Microporous Mesoporous Mater. 2009, 120 (3) 325-330DOI: 10.1016/j.micromeso.2008.11.020 
35.Lenk, T. J.; Hallmark, V. M.; Hoffmann, C. L.; Rabolt, J. F.; Castner, D. G.; Erdelen, C.; Ringsdorf, H. Structural Investigation of Molecular-Organization in SelfAssembled Monolayers of a Semifluorinated Amidethiol Langmuir 1994, 10 (12) 4610- 4617DOI: 10.1021/la00024a037

36.Chen, H.; Wang, L.; Yang, J.; Yang, R. T. Investigation on Hydrogenation of Metal-Organic Frameworks HKUST-1, MIL-53, and ZIF-8 by Hydrogen Spillover J. Phys. Chem. C 2013, 117 (15) 7565- 7576DOI: 10.1021/jp401367k

37.Biesinger, M. C.; Lau, L. W. M.; Gerson, A. R.; Smart, R. S. C. Resolving Surface Chemical States in XPS Analysis of First Row Transition Metals, Oxides and Hydroxides: Sc, Ti, V, Cu and Zn Appl. Surf. Sci. 2010, 257 (3) 887- 898DOI: 10.1016/j.apsusc.2010.07.086

38.Szelagowska-Kunstman, K.; Cyganik, P.; Goryl, M.; Zacher, D.; Puterova, Z.; Fischer, R. A.; Szymonski, M. Surface Structure of Metal-Organic Framework Grown on Self-Assembled Monolayers Revealed by High-Resolution Atomic Force Microscopy J. Am. Chem. Soc. 2008, 130 (44) 14446- 14447DOI: 10.1021/ja8069743

39.Kondo, M.; Furukawa, S.; Hirai, K.; Kitagawa, S. Coordinatively Immobilized Monolayers on Porous Coordination Polymer Crystals Angew. Chem., Int. Ed. 2010, 49 (31) 5327- 5330DOI: 10.1002/anie.201001063

40.Haemers, S.; Koper, G. J. M.; Frens, G. Effect of Oxidation Rate on Cross-Linking of Mussel Adhesive Proteins Biomacromolecules 2003, 4 (3) 632- 640DOI: $10.1021 / \mathrm{bm} 025707 \mathrm{n}$

41.Novio, F.; Ruiz-Molina, D. Hydrophobic Coordination Polymer Nanoparticles and Application for Oil-water Separation RSC Adv. 2014, 4 (29) 15293DOI: 10.1039/C4RA00850B

42.Saiz-Poseu, J.; Faraudo, J.; Figueras, A.; Alibes, R.; Busqué, F.; Ruiz-Molina, D. Switchable Self-Assembly of a Bioinspired Alkyl Catechol at a Solid/Liquid Interface: Competitive Interfacial, Noncovalent, and Solvent Interactions Chem. - Eur. J. 2012, 18 (10) 3056-3063DOI: 10.1002/chem.201101940 